\title{
Prevalencia de síntomas relacionados con el asma en escolares de Campos Gerais (MG), Brasil
}

\author{
Paulo A. M. Camargos, ${ }^{1}$ Roberta M. Castro ${ }^{2}$ y Juliana S. Feld- \\ $\operatorname{man}^{3}$
}

RESUMEN El asma es la enfermedad crónica más común en los niños y adolescentes y en los decenios más recientes se han registrado aumentos insólitos de su prevalencia en todas partes del mundo. Sin embargo, los estudios anteriores se han basado en distintos enfoques metodológicos, lo que dificulta hacer comparaciones entre los resultados. Ese factor estimuló a un grupo internacional de investigadores a elaborar un cuestionario estandarizado autoadministrable que permitiera llevar a cabo estudios internacionales en colaboración. Se creó así el instrumento utilizado en el International Study of Asthma and Allergies in Childhood (ISAAC) o Estudio Internacional del Asma y las Alergias en la Niñez. Durante el mes de abril de 1996 se realizó en la ciudad de Campos Gerais, Minas Gerais, Brasil, un estudio transversal para determinar la prevalencia en escolares del estertor sibilante y otras señales y sintomas relacionados con el asma bronquial utilizando el cuestionario elaborado para el ISAAC. Se seleccionaron de forma aleatoria simple 200 adolescentes de 13 a 14 años de edad matriculados en escuelas públicas del municipio, los cuales fueron entrevistados más tarde por dos estudiantes de medicina. La muestra estaba distribuida igualmente (50\%) por sexo y edad. Se encontró que $28,5 \%$ de los escolares (IC95\%: 22,3\% a 35,3\%) habian experimentado por lo menos un episodio de sibilancia en algún momento del pasado y que 6\% de ellos (IC95\%: 3,1 a 10,5\%) habían tenido por lo menos una crisis asmática en los últimos 12 meses. Al comparar esos resultados con los obtenidos en ciudades brasileñas de mayor tamaño, se observa un aumento de las tasas de prevalencia que parece corresponder al aumento en densidad de la población y al grado de urbanización e industrialización de los municipios estudiados. Se refuerzan así las teorías que sostienen la existencia de una asociación entre el asma y los factores ambientales. De ello se desprende que es necesario hacer frente a ese trastorno como importante problema de salud pública en el Brasil.

El asma es la enfermedad crónica más común en los niños y adolescentes $\mathrm{y}$, en consecuencia, la causa de elevados

\footnotetext{
Universidad Federal de Minas Gerais, Facultad de Medicina, Departamento de Pediatría. Toda correspondencia referente a este artículo debe enviarse a este autor a la siguiente dirección postal: Departamento de Pediatria, Faculdade de Medicina da Universidade Federal de Minas Gerais, Avenida Alfredo Balena, 190/Sala 4061, 30130-100 Belo Horizonte, MG, Brasil. Correo electrónico: pcamargs@medicina.ufmg.br

2 Fundación Hospitalaria del Estado de Minas Gerais, Brasil, Servicio de Pediatría.

3 Hospital de la Policía Militar de Minas Gerais, Brasil, Servicio de Clínica Médica.
}

costos sociales y económicos. En los decenios más recientes se han incrementado continuamente las tasas de prevalencia del asma, fenómeno que han observado en todas partes del mundo los clínicos, epidemiólogos y demás estudiosos de la enfermedad (1-4).

En Melbourne, Australia, por ejemplo, en el período de 1964 a 1990 se registró un aumento de $141 \%$ en la prevalencia del asma en los niños de 7 años de edad (1). Con un poco menos de intensidad, lo mismo se observó en el grupo de 8 a 13 años de edad en
Aberdeen, Escocia, donde la tasa casi se duplicó de 1964 a 1989 y pasó de 10,4\% a 19,8\% (2). En los Estados Unidos de América, el mismo indicador subió en el grupo de 6 a 11 años de edad, de 4,8\% en el período de 1971 a 1974 a 7,6\% en el de 1976 a 1980 (3). En estudios realizados en América Latina también se ha hecho evidente que de 5,7 a $16,5 \%$ de los niños y adolescentes tienen antecedentes de síntomas que sugieren asma (4).

No obstante, las tasas de prevalencia citadas se obtuvieron con enfoques me- 
todológicos diferentes, especialmente en lo que respecta al contenido del instrumento de recolección de datos, lo que pone en duda su validez externa $y$, en consecuencia, su comparabilidad con estudios similares. Este factor ampliamente conocido motivó a un grupo de investigadores de diversas nacionalidades a elaborar dos protocolos estandarizados para la recolección de datos - un cuestionario autoadministrable y un videocuestionario- dirigidos a llevar a cabo un estudio internacional en colaboración, que permitiera generar resultados partiendo de un instrumento común y elaborar un mapa mundial del asma para facilitar las comparaciones entre países y entre ciudades de un mismo país. Se constituyó, por lo tanto, un amplio grupo de trabajo multicéntrico para dirigir el Estudio Internacional de Asma y Alergia en la Infancia, más conocido por ISAAC, la sigla de International Study of Asthma and Allergies in Childhood, su nombre en inglés (5).

En la primera fase del ISAAC, participaron en el estudio cerca de 155 centros colaboradores localizados en 56 países $(6,7)$. Como primera de tres etapas, se entrevistaron alrededor de 700000 niños y adolescentes de los grupos de 6 a 7 y 13 a 14 años de edad para determinar la prevalencia y la intensidad de la rinitis alérgica y la eccema atópica, síntomas relacionados con el asma, en esos grupos (6). Como la mayoría de los investigadores y de los centros participantes estaban ubicados en ciudades de tamaño grande $o$ mediano, así fueron también los municipios investigados (6). Además, solo en conglomerados urbanos de esas dimensiones se podía lograr el tamaño muestral de 3000 personas que exigía el protocolo.

Por las razones expuestas, el componente brasileño del estudio - que abarcó siete municipios de las macrorregiones nordeste, sudeste y sur- incluyó las capitales de cinco unidades federativas (Recife, Pernambuco; Rio de Janeiro, Rio de Janeiro; São Paulo, São Paulo; Curitiba, Paraná; y Porto Alegre, Rio Grande do Sul) y dos ciudades importantes del interior del estado de Minas Gerais (Itabira y Uber- landia), donde la prevalencia de la sibilancia en niños de 13 a 14 años durante los 12 meses anteriores había oscilado entre 9,3 y $27 \%$ (6-8). En el ámbito internacional, en ese mismo grupo de edad se han obtenido tasas que varían, aproximadamente, desde $3 \%$ en Albania, China, Grecia, Georgia, Indonesia y Rusia hasta 30\% en Australia, Irlanda, Nueva Zelandia y el Reino Unido $(6,7)$.

En este estudio se desplaza el enfoque de las investigaciones hasta entonces realizadas con el cuestionario ISAAC (5) para procurar evaluar la prevalencia de los síntomas asociados con el asma en escolares de 13 a 14 años en una ciudad pequeña. Antes de usar el cuestionario, fue necesario hacer algunas adaptaciones indispensables del texto, el cual fue elaborado originalmente en inglés.

\section{MATERIALES Y MÉTODOS}

\section{Lugar y período de estudio}

Este estudio se llevó a cabo durante el mes de abril de 1996 en el municipio de Campos Gerais, situado a $21^{\circ}$ de latitud y $868 \mathrm{~m}$ de altitud en la región sur del estado de Minas Gerais. Durante el año la temperatura oscila entre un máximo de $30^{\circ} \mathrm{C}$ y un mínimo de $14^{\circ} \mathrm{C}$ con una media de $21^{\circ} \mathrm{C}$. Campos Gerais tiene una extensión de $641 \mathrm{~km}^{2}$ y una población de aproximadamente 24000 habitantes, la mitad de los cuales viven en la sede del municipio, lo que resulta en una densidad media de 40 habitantes por $\mathrm{km}^{2}$. El lugar es básicamente rural y casi $70 \%$ de la fuerza de trabajo se ocupa de la agricultura y la cría de ganado vacuno, que son las principales actividades económicas. El sector industrial emplea $9 \%$ de la población económicamente activa y el sector de los servicios, 17\%. La actividad industrial está limitada a microempresas que producen muebles, productos de limpieza, piezas de metal, bloques de cemento y artículos de ropa o se ocupan de la preparación y el empaquetado de arroz y café.

En general, la tasa de alfabetización llega a $75 \%$, pero alcanza $90 \%$ entre los niños de 10 a 14 años. La red de escuelas está constituida casi totalmente por instituciones públicas; las municipales instaladas en la zona rural y las estatales localizadas en la zona urbana, donde está matriculada $95 \%$ de la población de 5 a 14 años.

En la zona urbana del municipio, los servicios públicos de salud constan de una unidad de atención básica, un centro ambulatorio de atención primaria y secundaria y un pequeño hospital de unas 70 camas. La razón médico:habitante es de 1:1300, aproximadamente.

La red de distribución de agua potable sirve a más de $90 \%$ de los hogares urbanos y $98 \%$ de estos tienen alcantarillado propio.

\section{Población de estudio}

Para conocer a fondo el universo completo de adolescentes de 13 a 14 años matriculados en las escuelas públicas estatales del municipio, se revisaron los registros de las seis que estaban situadas en la sede del municipio y se realizó un censo minucioso de los alumnos de este grupo de edad que asistían a los ocho primeros grados. Se encontró una matrícula de 662 adolescentes, de los cuales se seleccionaron posteriormente 200 para integrar la muestra de estudio. La selección se llevó a cabo mediante números aleatorios generados por el paquete estadístico Epi Info, versión 6.04 (9).

\section{Contenido y aplicación del cuestionario}

Se usó el cuestionario básico propuesto por el ISAAC, el cual fue aprobado en Alemania en diciembre de 1991 en una reunión de trabajo en la que participaron especialistas internacionales (5). Para que se entendieran claramente las preguntas, el cuestionario se tradujo al portugués y se modificó de acuerdo con el lenguaje normalmente empleado por médicos y legos. Se compone de ocho preguntas, de las cuales tres intentan detectar las tasas de prevalencia acumulada (las preguntas 1 y 6) y actual (la pregunta 
2), es decir, los síntomas asociados con asma que se hubieran presentado en cualquier momento del pasado y en los últimos 12 meses, respectivamente. Las demás preguntas (número 3, 4, 5, 7 y 8) están destinadas a evaluar la intensidad de los síntomas (anexo 1).

Aunque la metodología original tenía prevista la autoadministración del cuestionario, este fue aplicado por dos estudiantes de último año de medicina, quienes emplearon las técnicas habituales de anamnesis en entrevistas individuales con los participantes. Sin embargo, los estudiantes se concentraron exclusiva y rigurosamente en el texto sin dar explicaciones adicionales.

\section{Aspectos estadísticos}

Tamaño muestral. El número de niños participantes se calculó según la fórmula clásica que se emplea para obtener los tamaños muestrales de estudios de corte transversal (10). Para ese fin se utilizaron los siguientes parámetros: 1) el universo de 662 alumnos de 13 a 14 años de edad disponible para el estudio; 2) la estimación de 5\% de asmáticos en ese universo, basada arbitrariamente en la prevalencia del asma entre los escolares de un país industrializado en los años setenta (3); y 3) un margen de error de $\pm 3 \%$ para la prevalencia encontrada; o sea, en el caso de que la tasa real fuera de $5 \%$, como se propone en el punto 2 , la variación del intervalo de confianza de 95\% tendría una amplitud de 6\%. En otras palabras, si se realizaran otras 100 investigaciones con el mismo objetivo y los mismos parámetros para el tamaño de la muestra, como el error alfa previamente aceptado es de $5 \%$, en $95 \%$ de ellas la tasa detectada estaría situada dentro del margen de error estipulado, es decir, la prevalencia podría variar de 2 a $8 \%$.

Según el cálculo realizado con el programa Epi Info, versión 6.04 (9), se necesitaba una muestra de 156 estudiantes. Suponiendo que $20 \%$ estarían ausentes de la escuela en la fecha programada, rehusarían participar o no responderían por otros motivos, el número de participantes se aumentó a
188, a los que se añadieron otros 12 para incrementar la precisión de las estimaciones. La muestra final se compuso de 200 escolares y, de ser adecuados los cálculos preliminares, era de esperar que se encontraran 10 niños asmáticos entre los entrevistados. Debe señalarse que, si bien esa muestra puede producir una estimación de la tasa de prevalencia, tiene menos precisión estadística para determinar los valores de intensidad de los síntomas. En este estudio, esos valores deben considerarse resultados secundarios.

\section{Banco de datos}

Se utilizó el formato original del banco de datos elaborado especialmente para el estudio colaborativo del ISAAC que, por su parte, también hizo uso de ciertos módulos del programa Epi Info (9).

\section{Pruebas estadísticas}

Se calcularon las distribuciones de frecuencias para caracterizar a la población estudiada y la sintomatología referida por los entrevistados, y el intervalo de confianza de $95 \%$ para obtener la variación de los valores.

\section{Aspectos éticos}

Los adolescentes que respondieron al cuestionario se dispusieron voluntariamente a participar en la investigación. La información que revelaron se considera rigurosamente confidencial y se presenta únicamente en forma de datos agregados.

\section{RESULTADOS}

Ninguno de los 200 escolares elegidos para formar la muestra rehusó responder al cuestionario. Sus características generales aparecen en el cuadro 1. Como resultado de la forma aleatoria de selección, hubo una distribución equitativa por sexo y edad. Aun así puede observarse que un número pe- queño de adolescentes asistía a los primeros cuatro grados de primaria (44/200, o sea $22 \%$ ). El resto (78\%) estaba distribuido entre los demás grados. El cuadro 1 indica también que en la muestra se incluyeron todas las seis escuelas estatales ubicadas en la sede del municipio.

En cuanto a la prevalencia e intensidad de los síntomas asociados con el asma, el cuadro 2 muestra los resultados obtenidos aplicando el protocolo estandarizado propuesto para el ISAAC (véase el anexo 1). Se destaca que 57 de los 200 adolescentes entrevistados $(28,5 \%)$ relataron que habían tenido por lo menos un episodio de sibilancia en el pasado (prevalencia acumulada). Entre el límite inferior $(22,3 \%)$ y el superior $(35,3 \%)$ del intervalo de confianza de 95\% (IC95\%) hay una diferencia de $13 \%$, que es el margen de error de la estimación. De estos alumnos, 30 eran varones $(52,6 \%)$ y 27 , mujeres $(47,4 \%)$.

La epidemiología y la historia natural del asma permiten presumir que la tasa de prevalencia actual debe ser menor que la de prevalencia acumulada. Esto se confirmó en nuestro estudio ya que, de los 57 alumnos que respondieron afirmativamente a la pregunta anterior, $45(78,9 \%)$ no habían tenido episodios de sibilancia en los 12 meses anteriores, pero los 12 adolescentes restantes $(6 \%$ del total de encuestados) sí los habían tenido. De estos, 8 eran niñas $(66,7 \%)$ y 4 , niños $(33,3 \%)$. La tasa estimada correspondiente es la prevalencia actual del asma en la población estudiada. La variación estimada de esta tasa es de IC95\%: (3,1\% a 10,5\%). La amplitud de la variación $(7,4 \%)$ se acerca al margen de error de $6 \%$ supuesto para calcular el tamaño de la muestra.

De los 12 adolescentes que tuvieron sibilancia en los 12 meses anteriores, 10 (83,3\%) (IC95\%: 51,6\% a 97,8\%) dijeron haber tenido de una a tres crisis durante ese período. En los restantes $(16,7 \%)$ (IC95\%: 2,1\% a 48,4\%), los episodios fueron más frecuentes. Además, durante las crisis agudas de los 12 meses anteriores, cinco (41,7\%) de los 12 niños (IC95\%: 15,1\% a 72,3\%) habían sufrido interrupción del sueño 
CUADRO 1. Características generales de 200 adolescentes entrevistados sobre la frecuencia de síntomas asociados con el asma, Campos Gerais (MG), Brasil, abril de 1996

\begin{tabular}{|c|c|c|}
\hline Variable & No. & $\%$ \\
\hline \multicolumn{3}{|l|}{ Sexo } \\
\hline Masculino & 100 & 50,0 \\
\hline Femenino & 100 & 50,0 \\
\hline Total & 200 & 100,0 \\
\hline \multicolumn{3}{|l|}{ Grupo de edad } \\
\hline 13 años cumplidos & 100 & 50,0 \\
\hline 14 años cumplidos & 100 & 50,0 \\
\hline Total & 200 & 100,0 \\
\hline \multicolumn{3}{|l|}{ Grado escolar } \\
\hline Primero & 10 & 5,0 \\
\hline Segundo & 2 & 1,0 \\
\hline Tercero & 18 & 9,0 \\
\hline Cuarto & 14 & 7,0 \\
\hline Quinto & 38 & 19,0 \\
\hline Sexto & 35 & 17,5 \\
\hline Séptimo & 46 & 23,0 \\
\hline Octavo & 37 & 18,5 \\
\hline Total & 200 & 100,0 \\
\hline \multicolumn{3}{|l|}{ Institución } \\
\hline Escuela Estatal Prof. Eduardo Dias & 104 & 52,0 \\
\hline Escuela Estatal Monseñor Teófilo Saez & 60 & 30,0 \\
\hline Escuela Estatal Carlos Góes & 22 & 11,0 \\
\hline Escuela Estatal João Barbalho & 7 & 3,5 \\
\hline Escuela Estatal José Augusto de Mesquita & 4 & 2,0 \\
\hline Escuela Estatal Joaquim José de Oliveira & 3 & 1,5 \\
\hline Total & 200 & 100,0 \\
\hline
\end{tabular}

una o más veces a la semana y siete (58,3\%) (IC95\%: 27,6\% a 84,8\%), disnea al mínimo esfuerzo, por ejemplo, al hablar (véanse las preguntas 4 y 5 del anexo 1.)

La pregunta número 6 se formuló intencionalmente para comprobar su consonancia con las respuestas a la primera pregunta. Se ve que la proporción de respuestas afirmativas $(27 \%)$ es similar a la de la primera pregunta $(28,5 \%)$. No obstante, es preciso resaltar que entre los 57 y 54 escolares que respondieron afirmativamente a las preguntas 1 y 6 , respectivamente, 36 mostraron concordancia parcial $(65 \%)$.

Las dos respuestas finales indican que, en los 12 meses anteriores, 24 $(12 \%)$ y $52(26 \%)$ de los 200 escolares entrevistados habían experimentado, respectivamente, otras manifestaciones asociadas con el asma, como sibilancia provocada por el ejercicio y tos seca nocturna.

\section{DISCUSIÓN}

Por la elaboración y administración de un instrumento de recolección de datos estandarizado y de alcance mundial, entre otras razones, el ISAAC puede considerarse uno de los estudios multicéntricos de base poblacional más importantes hasta ahora realizados. Sin estar integrado a la red compuesta por centros colaboradores, el estudio aquí presentado se llevó a cabo con la intención de explorar un aspecto no previsto originalmente en la metodología del ISAAC, o sea, verificar la tasa de prevalencia de una ciudad pequeña con características rurales.

Sin embargo, como suele ocurrir aun en investigaciones de la envergadura del ISAAC, el presente estudio no es inmune a sesgos. Estos pueden estar relacionados con el lenguaje, por la traducción del cuestionario al portugués, y con la cultura, en el sentido de cómo se perciben una enfermedad y sus sín- tomas, e incluso con el grado de escolaridad. Dados los posibles sesgos, es esencial tener cautela al establecer comparaciones. Además debe tenerse en cuenta que, contrario a la recomendación metodológica de la autoadministración del cuestionario adoptada en el estudio multicéntrico, en nuestro estudio se optó por aplicar el cuestionario en entrevistas individuales, hecho que puede haber añadido otro tipo de sesgo a los anteriores. Por otra parte, los resultados obtenidos se aplican solo a una población de adolescentes del área central de Campos Gerais debido a que, por cuestiones operacionales y financieras, las escuelas situadas en el área periférica del municipio quedaron excluidas del planeamiento de la muestra.

Si bien en el análisis y la discusión de los resultados debe considerarse la posible interferencia de los factores arriba mencionados, es posible que la participación total de los 200 escolares seleccionados para la muestra haya podido reducir, por lo menos en parte, esas influencias. Para el conjunto del ISAAC, la tasa de rechazo fue de menos de $15 \%$, pero pocos centros lograron una participación total $(6,7)$.

\section{Tasas de prevalencia}

Basada en las respuestas a las preguntas 1 a 6 del cuestionario (véanse el anexo 1 y el cuadro 2), la prevalencia acumulada de síntomas relacionados con el asma que se obtuvo en este estudio se situó entre 27 y $28,5 \%$, valores apenas superados por los de las ciudades de Adelaida (30,4\%) y Perth $(30,2 \%)$ en Australia, donde se registran las tasas más altas del mundo (6, 7). Al hacer una comparación con los resultados de las ciudades brasileñas que integraron el ISAAC, llama la atención que en ellas la proporción de adolescentes con antecedentes de asma (pregunta número 6) varió de $4,8 \%$ en Itabira a $21,8 \%$ en Porto Alegre (6-8), cifras menores que la tasa de $27 \%$ registrada en Campos Gerais.

Esa disparidad se manifiesta también al comparar las prevalencias acumulada y actual que encontramos en este estudio. Las encuestas epidemio- 
CUADRO 2. Prevalencia e intensidad de sibilancia asmática en 200 adolescentes estudiados, Campos Gerais (MG), Brasil, abril de 1996

\begin{tabular}{|c|c|c|c|}
\hline Pregunta formulada & No. & $\%$ & $\operatorname{IC} 95 \%{ }^{\mathrm{a}}$ \\
\hline \multicolumn{4}{|l|}{$\begin{array}{l}\text { 1. ¿Has tenido silbidos en el pecho } \\
\text { alguna vez en tu vida? }\end{array}$} \\
\hline Sí & 57 & 28,5 & 22,3 a 35,3 \\
\hline No & 135 & 67,5 & 60,5 a 73,9 \\
\hline No recuerda & 8 & 4,0 & - \\
\hline Total & 200 & 100,0 & - \\
\hline \multicolumn{4}{|l|}{$\begin{array}{l}\text { 2. ¿Has tenido silbidos en el pecho } \\
\text { en los últimos } 12 \text { meses? }\end{array}$} \\
\hline Sí & 12 & 6,0 & 3,1 a 10,5 \\
\hline No & 188 & 94,0 & 89,7 a 96,8 \\
\hline Total & 200 & 100,0 & - \\
\hline \multicolumn{4}{|c|}{$\begin{array}{l}\text { 3. ¿Cuántas veces has tenido silbidos en el } \\
\text { pecho en los últimos } 12 \text { meses?b }\end{array}$} \\
\hline Entre 1 y 3 veces & 10 & 83,3 & 51,6 a 97,8 \\
\hline Entre 4 y 12 veces & 2 & 16,7 & 2,1 a 48,4 \\
\hline Total & 12 & 100,0 & - \\
\hline \multicolumn{4}{|c|}{$\begin{array}{l}\text { 4. En los últimos } 12 \text { meses, ¿cuántas veces } \\
\text { en promedio se te ha interrumpido el sueño } \\
\text { por crisis de silbidos en el pecho?b }\end{array}$} \\
\hline Ninguna vez & 3 & 25,0 & 5,5 a 57,2 \\
\hline Menos de una vez a la semana & 4 & 33,3 & 9,9 a 65,1 \\
\hline Una o más veces a la semana & 5 & 41,7 & 15,1 a 72,3 \\
\hline Total & 12 & 100,0 & - \\
\hline \multicolumn{4}{|c|}{$\begin{array}{l}\text { 5. Las crisis de los últimos } 12 \text { meses, } \\
\text { ¿han sido tan graves que no has podido } \\
\text { hablar por falta de aire? }\end{array}$} \\
\hline Sí & 7 & 58,3 & 27,6 a 84,8 \\
\hline No & 5 & 41,7 & 15,1 a 72,3 \\
\hline Total & 12 & 100,0 & - \\
\hline \multicolumn{4}{|c|}{ 6. ¿Has tenido asma o bronquitis alguna vez? } \\
\hline Sí & 54 & 27,0 & 20,9 a 33,7 \\
\hline No & 138 & 69,0 & 62,0 a 75,3 \\
\hline No recuerda & 8 & 4,0 & - \\
\hline Total & 200 & 100,0 & - \\
\hline \multicolumn{4}{|c|}{$\begin{array}{l}\text { 7. En los últimos } 12 \text { meses, ¿te han dado } \\
\text { silbidos en el pecho al hacer ejercicio físico? }\end{array}$} \\
\hline Sí & 24 & 12,0 & 7,8 a 17,3 \\
\hline No & 174 & 87,0 & 81,5 a 91,3 \\
\hline No recuerda & 2 & 1,0 & - \\
\hline Total & 200 & 100,0 & - \\
\hline \multicolumn{4}{|c|}{$\begin{array}{l}\text { 8. En los últimos } 12 \text { meses, ¿has tenido } \\
\text { tos seca de noche sin estar resfriado ni tener } \\
\text { alguna infección respiratoria? }\end{array}$} \\
\hline Sí & 52 & 26,0 & 20,0 a 32,6 \\
\hline No & 147 & 73,5 & 66,8 a 79,4 \\
\hline No recuerda & 1 & 0,5 & - \\
\hline Total & 200 & 100,0 & - \\
\hline
\end{tabular}

a Intervalo de confianza de $95 \%$.

${ }^{b}$ Pregunta dirigida solamente a los adolescentes que respondieron afirmativamente a la segunda pregunta (véase el anexo 1).

lógicas hacen constar que al llegar a la adolescencia, en 30 a $50 \%$ de los pacientes han desaparecido las manifestaciones clínicas que tuvieron durante la infancia (11). Esa tendencia se manifiesta en el presente estudio, pero su magnitud se encuentra muy sobrestimada, pues en ocasiones hay una diferencia de 4,5 veces entre las tasas acumulada y actual. Dentro del contexto del ISAAC, tampoco hubo una excelente correlación entre las prevalencias actual y acumulada $(R=0,76)$, lo que muestra que en algunos países los adolescentes investigados declaran haber tenido sibilancia con mayor frecuencia en los 12 meses anteriores que en cualquier otro momento del pasado y viceversa $(6,7)$.

En realidad, esas cifras sugieren que hay un sesgo de recuerdo, puesto que es más fácil recordar los episodios que han ocurrido de 12 meses a esta parte que los de toda una vida. Esta hipótesis se ve reforzada por el grado de concordancia (65\%) entre las preguntas 1 y 6 del cuestionario. Es, pues, necesario interpretar de forma crítica esos resultados, ya que hay fuertes indicios de que ninguna de las dos preguntas es adecuada para fines epidemiológicos. Con esa salvedad, en los resultados que obtuvimos para la prevalencia actual -objetivo principal del presente estudio- parece haber menos interferencia, por lo que se prestan para análisis comparativos de las tasas obtenidas.

En la parte del ISAAC realizada en el Brasil, las ciudades grandes y medianas que se estudiaron fueron Curitiba, Itabira, Porto Alegre, Recife, Salvador, São Paulo y Uberlandia (6-8). La prevalencia de sibilancia en los 12 meses anteriores fue, en todos los casos, superior al $6 \%$ registrado en Campos Gerais; es decir, 18,4\%, 9,3\%, $24,6 \%$, 19,7\%, 27,1\% 23,3\% y 21,0\%, respectivamente (6-8).

La variación que se observa lleva a suponer que la prevalencia actual depende de la densidad de la población o del grado de urbanización del municipio estudiado. Comparando los resultados obtenidos en las distintas ciudades, se observa que las tasas disminuyen en los municipios con economía agropecuaria localizados en zonas rurales tales como Campos Gerais y se elevan en las megalópolis como São Paulo $(23,3 \%)$ y Salvador $(27,1 \%)$, siendo esta última la tercera ciudad del Brasil en cuanto a población. Se encontró un valor intermedio en Itabira $(9,3 \%)$, ciudad de tamaño mediano. Para explicar estas variaciones, deben investigarse los factores ambientales en el sentido más amplio de la palabra, se relacionen o no con los índices de 
contaminación atmosférica. Cabe notar que en Chile se registró una situación similar y la tasa verificada en Punta Arenas (6,8\%) fue más baja que la de las zonas central $(10,2 \%)$ y sur $(11,1 \%)$ de Santiago $(6,7)$. En general, la tasa de prevalencia actual en Campos Gerais es menor que las tasas medias obtenidas en ciudades más populosas tanto de América Latina como de los Estados Unidos de América y el Canadá, donde variaron de 16,9\% a 24,2\% $(6,7)$.

Por otra parte, considerando la amplitud de variación del IC95\% (3,1\% a $10,5 \%$ ) de la prevalencia de los síntomas asociados con el asma en los 12 meses anteriores en Campos Gerais, sorprende comprobar que están comprendidas dentro de la misma variación las tasas registradas en algunas ciudades grandes y medianas de España, Grecia, Italia y Portugal $(6,7)$. Ello hace pensar que acaso el grado de urbanización y el estado de industrialización no son suficientes para explicar la tasa relativamente más elevada verificada en un municipio de características rurales como es Campos Gerais o que quizá el protocolo del ISAAC sea más útil para hacer comparaciones dentro de un país que entre distintas naciones.

\section{Intensidad de los síntomas}

En lo que se refiere a estimar la frecuencia y la gravedad de los síntomas asociados con el asma, es preciso ser doblemente cauteloso, ya que el tamaño muestral, calculado para determinar la prevalencia actual, no es suficiente para ubicar esos parámetros dentro de límites estadísticamente aceptables. Esta ponderación puede ser fácilmente comprobada analizando la amplitud de la variación del IC95\% correspondiente a las respuestas dadas a las preguntas número 3, 4, 5, 7 y 8 (véase el cuadro 2). Además, la imprecisión de las estimaciones reduce mucho la posibilidad de compararlas con las tasas registradas en otros lugares que participaron en el estudio multicéntrico (6).

Aunque en el medio brasileño la prevalencia estimada de síntomas asociados con el asma en los 12 meses anteriores coincide con las tasas registradas en siete otras ciudades, las proporciones son muy dispares en cuanto a la gravedad de los síntomas (6). Ninguno de los valores registrados en Campos Gerais se aproxima a ellos y hay una clara tendencia a sobrestimar la intensidad del cuadro clínico del asma.

\section{Consideraciones finales}

La prevalencia actual de los síntomas asociados con el asma en la ciudad de Campos Gerais indica sin lugar a dudas que esta enfermedad es un problema de salud pública importante en el Brasil. Los resultados de este estudio han demostrado que aun en municipios menos populosos y con caracterís- ticas típicamente rurales se registran tasas considerables de esos síntomas.

La tendencia al aumento de la prevalencia de los síntomas asociados con el asma en el período anterior de 12 meses en adolescentes de 13 a 14 años se corresponde con el tamaño y grado de urbanización del municipio donde viven. Este hallazgo sienta algunas bases para la investigación futura, especialmente en relación con el papel que desempeñan los factores causales —ambientales, genéticos, infecciosos y conductuales - en la génesis de esta enfermedad en el Brasil. Estudios de ese tipo tendrían incluso implicaciones prácticas para la salud individual y colectiva en el sentido de hallar formas de prevenir el asma.

A la inversa, también es interesante comprobar que la amplitud de la variación del IC95\% de la tasa detectada en Campos Gerais (6\%) comprende valores encontrados en ciudades europeas con mayor urbanización, densidad poblacional, industrialización y contaminación atmosférica. Es posible que ello indique que, en el Brasil y otros lugares, la contaminación atmosférica de por sí no eleva demasiado el riesgo de desarrollar asma bronquial, aunque esta puede ser agravada por esas condiciones (7).

Agradecimiento. Los autores agradecen a la Prefectura Municipal de Campos Gerais, a las directoras y profesoras de las escuelas involucradas y a los alumnos participantes la colaboración prestada durante este estudio.

\section{REFERENCIAS}

1. Robertson CF, Heycock E, Bishop J, Nolan T, Olinsky A, Phelan PD. Prevalence of asthma in Melbourne schoolchildren: changes over 26 years. BMJ 1991;302:1116-1118.

2. Ninan TK. Russell G. Respiratory symptoms and atopy in Aberdeen schoolchildren: evidence from two surveys 25 years apart. BMJ 1992; 304:873-875.

3. Gergen PJ, Mullally DI, Evans R. 3rd. National survey of prevalence of asthma among children in the United States, 1976 to 1980. Pediatrics 1988;81:1-7.
4. Baeza Bacab MA, Albertos Alpuche NE. Prevalencia de asma en niños escolares de Mérida, Yucatán. Rev Panam Salud Publica 1997;2:299-302.

5. Asher MI, Keil U, Anderson HF, Beasley R, Crane J, Martínez F, et al. International study of asthma and allergies in childhood (ISAAC): rationale and methods. Eur Respir J 1995;8: 483-491.

6. The International Study of Asthma and Allergies in Childhood (ISAAC) Steering Committee. Worldwide variations in the prevalence of asthma symptoms: the International Study of Asthma and Allergies in Childhood (ISAAC). Eur Respir J 1998;12:315-335.

7. The International Study of Asthma and Allergies in Childhood (ISAAC) Steering Committee. Worldwide variations in prevalence of symptoms of asthma, allergic rhinoconjunctivitis, and atopic eczema: ISAAC. Lancet 1998;351:1225-1232.

8. Vana AT, Bezerra PGM, Calil J, Ferrari F, Jones M, Maspitz CK, et al. Prevalence of asthma in Brazilian teenagers: ISAAC study: 
International Pediatric Respiratory Congress, Sydney, New South Wales, Australia, March 16-19, 1997. P. 40.

9. Dean AG, Dean JA, Coulombier D, Burton AH, Brendel KA, Smith DC, et al. Epi Info, version 6.04: a word processing, database, and statistics program for public health on microcomputers. Centers for Disease Control and Prevention-World Health Organization; 1996.
10. Browner WS, Black D, Newman TB, Hulley SB. Estimating sample size and power. En: Hulley SB, Cummings SR, eds. Designing clinical research. Baltimore: Williams and Wilkins; 1988. P. 145.

11. Global Initiative for Asthma (GINA). Epidemiology. En: Global Strategy for Asthma Management and Prevention: report of a project in collaboration with the National Heart, Lung, and Blood Institute, NIH, and the World Health Organization. Bethesda, Maryland: NIH/NHLBI —WHO; 1995. Pp. 10-22.

Manuscrito recibido el 21 de junio de 1998 y aceptado para publicación en versión revisada el 2 de marzo de 1999.

ANEXO 1. Cuestionario estandarizado propuesto por el Estudio Internacional del Asma y las Alergias en la Niñez (ISAAC)

Identificación del entrevistado

Grado escolar

Primero $\quad$ Segundo $\quad$ Tercero $\quad$ Cuarto

Escuela

\section{Prof. Eduardo Dias} João Barbalho
Monseñor Teófilo Saez
José Augusto de Mesquita

Carlos Góes

Joaquim José de Oliveira

Fecha de la entrevista

Edad

13 años cumplidos 14 años cumplidos

Fecha de nacimiento

Sexo

Masculino Femenino

1. ¿Has tenido silbidos en el pecho alguna vez en tu vida?
Sí
No
No recuerda

[Si la respuesta es negativa, ir directamente a la pregunta número 6]

2. ¿Has tenido silbidos en el pecho en los últimos 12 meses?

Sí

No

[Si la respuesta es negativa, ir directamente a la pregunta número 6]

3. ¿Cuántas veces has tenido silbidos en el pecho en los últimos 12 meses?

Ninguna Entre 1 y 3 veces Entre 4 y 12 veces Más de 12 veces

4. En los últimos 12 meses, ¿cuántas veces en promedio se te ha interrumpido el sueño por crisis de silbidos en el pecho?

Ninguna Menos de una vez a la semana o más veces a la semana

5. Las crisis de los últimos 12 meses ¿han sido tan graves que no has podido hablar por falta de aire? Sí

No

6. ¿Has tenido asma o bronquitis alguna vez?
Sí
No
No recuerda

7. En los últimos 12 meses, ¿te han dado silbidos en el pecho al hacer ejercicio físico?
Sí
No
No recuerda

8. En los últimos 12 meses, ¿has tenido tos seca de noche sin estar resfriado ni tener alguna infección respiratoria?

$$
\text { Sí }
$$

No

No recuerda

Fuente: Referencia 5. 
ABSTRACT Asthma is the most common chronic disease among children and adolescents, and in recent decades dramatic increases in its prevalence have been reported around the world. However, previous studies have been based on a variety of methodological approaches, making it difficult to compare the results. That situation led a group of international researchers to prepare a standardized self-administered questionnaire to carry out cooperative international studies for the International Study of Asthma and Allergies in Childhood (ISAAC). During April 1996 a cross-sectional study using the ISAAC questionnaire was performed in the city of Campos Gerais, Minas Gerais, Brazil, to determine the prevalence of wheezing and other signs and symptoms related to bronchial asthma among schoolchildren. Two medical students interviewed a randomly selected sample of 200 adolescents 13-14 years old registered in public schools in the city. The sample was equally distributed by sex and age. Of the schoolchildren, $28.5 \%$ (95\% CI: $22.3 \%-35.3 \%$ ) had had wheezing or whistling in the chest at some point in the past. In the preceding 12 months, $6 \%$ of them (95\% CI: $3.1 \%-10.5 \%)$ had had at least one wheezing episode. A comparison of these results with ones from larger Brazilian cities studied showed that higher prevalence rates were associated with higher population densities and greater urbanization and industrialization. These results strengthen theories supporting an association between asthma and environmental factors. From this it follows that it is necessary to deal with asthma as an important public health problem in Brazil.

\begin{tabular}{|c|c|}
\hline \multicolumn{2}{|c|}{$\begin{array}{c}\text { Sexto Congreso Anual de la International Society for } \\
\text { Quality of Life Research (ISOQOL) }\end{array}$} \\
\hline $\begin{array}{l}\text { Fechas: } \\
\text { Lugar: } \\
\text { Tema: }\end{array}$ & $\begin{array}{l}3 \text { a } 6 \text { de noviembre de } 1999 \\
\text { Barcelona, España } \\
\text { Measurement, Valuation and Interpretation of } \\
\text { Changes in Health-Related Quality of Life: } \\
\text { Time for a Critical Review }\end{array}$ \\
\hline $\begin{array}{l}\text { En las fechas indicadas, la } \\
\text { Seattle, Washington, Estados U } \\
\text { Barcelona, España. El primer día } \\
\text { pañola de Calidad de Vida Relac } \\
\text { ambos eventos gravitarán en torn } \\
\text { calidad de vida en relación con la } \\
\text { evaluación de la calidad de vida } \\
\text { de ensayos clínicos y del ejercicio } \\
\text { pectos de la determinación de la }\end{array}$ & 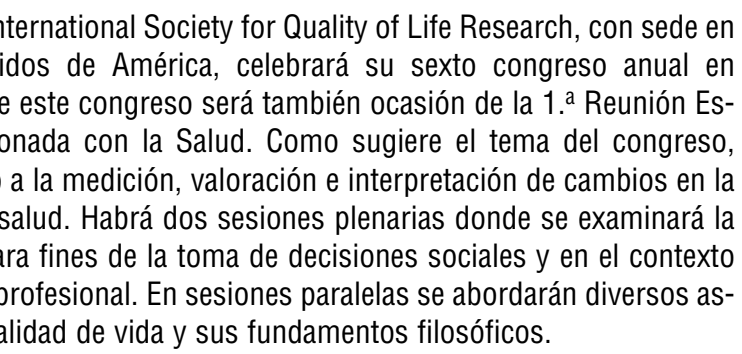 \\
\hline Correo & $\begin{array}{l}\text { Información: } \\
\text { retaría de Organización: RCT } \\
\text { Aulestia i Pijoan, } 12 \text { baixos } \\
\text {-08012 Barcelona, España } \\
\text { Tel.: +34-93-415 } 6938 \\
\text { Fax: +34-93-415 } 6904 \\
\text { ectrónico: ret@ret-congresos.com }\end{array}$ \\
\hline
\end{tabular}

